Abstract

\title{
Dissipative Extension of Electrodynamics ${ }^{\dagger}$
}

\section{Peter Ván}

MTA Wigner Research Centre for Physics, Budapest, Hungary

+ Presented at the Entropy 2021: The Scientific Tool of the 21st Century, 5-7 May 2021; Available online: https://sciforum.net/conference/Entropy2021/.

Published: 5 May 2021

In nonequilibrium thermodynamics, electrodynamic interaction and electrodynamic forces appear as non-dissipative, external phenomena. Irreversibility is due to Ohm's law and polarisation. However, the theoretical approaches of polarisation and thermal couplings do not apply to Lorentz force and electromagnetic stresses. The choice of state variables is also problematic. Thermodynamic stability cannot be valid for para- and diamagnetic materials at the same time, choosing either magnetisation or the magnetic field strength, or the corresponding four quantities in a special relativistic framework, as a state variable. Moreover, any particular choice leads to shape-dependent homogeneous thermodynamic bodies, and therefore the extensivity condition of thermodynamic state variables cannot be introduced without any further ado. In the presentation, I survey the problems of thermodynamic compatibility of electrodynamics and suggest some explanations. The main ideas are originated in a novel approach to gravity in the framework of nonequilibrium thermodynamics, where the gravitational potential is a thermodynamic state variable, and the balances of mass, momentum and energy are constraints for the entropy inequality. Naturally, for electrodynamics, special relativity is a necessary background.

(C) 2021 by the author. Licensee MDPI, Basel, Switzerland. This article is an open access article distributed under the terms and conditions of the Creative Commons Attribution (CC BY) license (http://creativecommons.org/licenses/by/4.0/). 